\title{
Effects of febuxostat and inosine on rat myocardial ischemia/reperfusion injury
}

\author{
Takako Yao ${ }^{1}$ and Yoshinori Seko ${ }^{2 *}$ \\ ${ }^{1}$ Division of Cardiovascular Medicine, The Institute for Adult Diseases, Asahi Life Foundation, Tokyo, Japan \\ ${ }^{2}$ Department of Biofunctional Microbiota, Juntendo University School of Medicine, Tokyo, Japan
}

\begin{abstract}
Adenosine triphosphate (ATP) is the source of energy for various organs especially the heart muscle. ATP degradation pathways reach to uric acid through hypoxanthine and xanthine as the last two steps, which are catalyzed by xanthine oxidase (XO). Treatment with XO inhibitor has been shown to increase myocardial mechanical efficiency and improves cardiac contractility and myocardial ischemia. The purpose of this study is to investigate whether treatment with an XO inhibitor febuxostat along with a nucleoside inosine, upstream of hypoxanthine, can have protective effects on rat myocardial ischemia/reperfusion injury. Treatment with both febuxostat alone and (febuxostat plus inosine) significantly reduced the infarct size $(23.24 \pm 1.89 \%$ and $22.34 \pm 2.28 \%$, respectively; $\mathrm{n}=5$ each) as compared with the control treatment $\left(34.40 \pm 2.97 \%, n=5,{ }^{*} \mathrm{P}<0.0174\right.$ and ${ }^{* *} \mathrm{P}<0.0109$, respectively). However, there was no significant difference between febuxostat-treated and (febuxostat plus inosine)-treated groups. Because XO enzyme activities in rodents were reported to be 100 times higher than those in humans, XO inhibition alone may supply enough materials for ATP production in rodents. We think there is a possibility that combination therapy with febuxostat plus inosine will be more effective than febuxostat alone in reducing the myocardial ischemia/reperfusion injury in humans, and further clinical investigation is needed to verify this.
\end{abstract}

\section{Introduction}

Because cardiac myocytes need to repeat contraction and relaxation continuously, they demand a lot of energy that is usually supplied as adenosine triphosphate (ATP). ATP is made by oxidative phosphorylation from adenosine diphosphate (ADP) in the mitochondria. The ATP breakdown pathways below ADP are known to reach uric acid through hypoxanthine and xanthine, in which the last two steps are catalyzed by an enzyme xanthine oxidase (XO). XO also plays a critical role in producing reactive oxygen species (ROS), which inhibit creatine kinase $(\mathrm{CK})$ reaction transferring mitochondrial energy to the cytosolic contractile system. Therefore, it is proposed that inhibition of XO may increase hypoxanthine as a source of ATP as well as enhance the energy transfer for contraction [1]. Evidence has accumulated that treatment with $\mathrm{XO}$ inhibitor such as allopurinol increases myocardial mechanical efficiency and improves cardiac contractility in the animal models of heart failure [2-4] and in patients with heart failure [5-7]. In fact, allopurinol significantly prolonged the exercise time to occur myocardial ischemia in patients with angina pectoris [8]. It has been reported that another $\mathrm{XO}$ inhibitor febuxostat is more effective than allopurinol in reducing uric acid levels as well as improving cardiac contraction in patients with heart failure [9]. A nucleoside, inosine is an intermediate metabolite of ATP breakdown pathway upstream of hypoxanthine is also a material of ATP synthesis. Inosine has ever been reported to act as a coronary vasodilator as well as an inotropic agent $[10,11]$, although controversial $[12,13]$. The purpose of this study was to investigate whether $\mathrm{XO}$ inhibitor febuxostat can have protective effects on a rat model of myocardial ischemia/reperfusion injury and whether inosine administration has additive effects.

\section{Methods}

\section{Febuxostat and Inosine administration}

Sprague Dawley (SD) rats (male, $200 \mathrm{~g}$ ) were used in this study. The rats were divided into control group, febuxostat $(5 \mathrm{mg} / \mathrm{Kg})$-treated group, and (febuxostat $[5 \mathrm{mg} / \mathrm{Kg}]$ plus inosine $[375 \mathrm{mg} / \mathrm{Kg}]$ )-treated group. Febuxostat and inosine were dissolved in dimethyl sulfoxide and water, respectively. Rats received febuxostat/inosine orally 1 hour before the start of ischemia.

\section{Myocardial ischemia/reperfusion}

The procedures for myocardial ischemia/reperfusion were performed as described elsewhere [14]. The rats were euthanized 24 $\mathrm{h}$ after reperfusion, and the rats that died before $24 \mathrm{~h}$ of reperfusion were excluded from the analysis. All animals were randomized to the control, febuxostat-treatment, and (febuxostat plus inosine)-treatment groups. After $24 \mathrm{~h}$ of reperfusion, the left ventricle of each heart was excised and cut transversely into six sections from the apex to the base. The tissue samples were then incubated in $1 \%$ triphenyl tetrazolium chloride (TTC) solution buffered in $0.2 \mathrm{~mol} / \mathrm{L}$ Tris buffer. The infarct

Correspondence to: Yoshinori Seko, M.D., Ph.D., Department of Biofunctional Microbiota, Juntendo University School of Medicine, 2-1-1 Hongo, Bunkyo-ku, Tokyo 113-8421, Japan, Tel: +81-3-5802-1591; Fax: +81-3-3813-5512; E-mail: sekoyosh-tky@umin.ac.jp

Key words: adenosine triphosphate (ATP), febuxostat, ischemia/reperfusion injury, inosine, xanthine oxidase (XO)

Received: May 27, 2017; Accepted: June 13, 2017; Published: June 15, 2017 
A

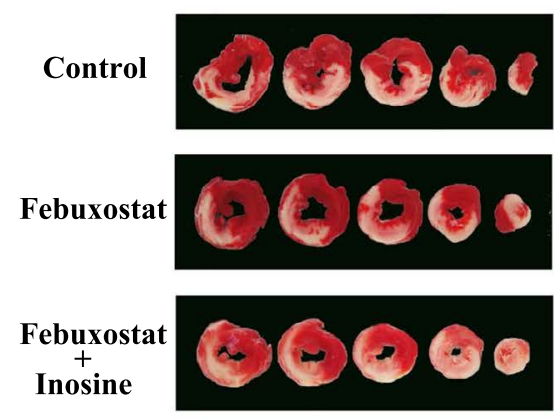

B

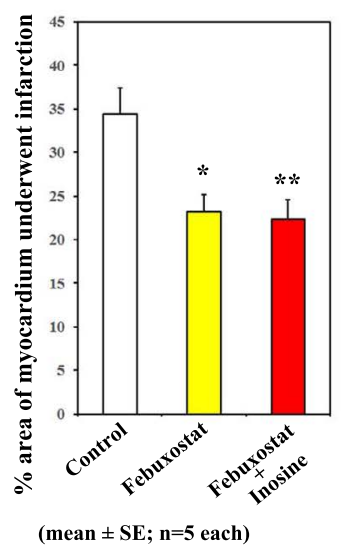

Figure 1. Febuxostat treatment suppresses myocardial ischemia/reperfusion injury in vivo. A) Representative cross-sections of a heart from the control group (upper panel), the febuxostattreated group (middle panel), and the (febuxostat plus inosine)-treated group (lower panel). B) The infarct size in the febuxostat-treated group and the (febuxostat plus inosine)-treated groups were significantly smaller than that in the control group ( $\mathrm{n}=5$ each, $* P<0.0174$ and $* * P<0.0109$, respectively), however, there was no significant difference between the febuxostat-treated group and the (febuxostat plus inosine)-treated groups $(P=0.963$; Tukey-Kramer method).

size was then determined by adding all of the area of infarction for each left ventricle. Infarct size was expressed as a percentage of left ventricle. All animal experiments were performed in accordance with the Guide of The Japanese Association of Laboratory Animal Facilities of National University Corporations. Statistical analysis was conducted by Tukey-Kramer method for multiple comparison test.

\section{Results}

As shown in Figure 1 (panels A and B), treatment with either febuxostat only or (febuxostat plus inosine) significantly reduced the infarct size $(23.24 \pm 1.89 \%$ and $22.34 \pm 2.28 \%$, respectively; $\mathrm{n}=5$ each) as compared with the control treatment $(34.40 \pm 2.97 \%, n=5$, ${ }^{\star} P<0.0174$ and ${ }^{*} P<0.0109$, respectively). However, there was no significant difference between febuxostat-treated and (febuxostat plus inosine)-treated groups $(P=0.963)$.

\section{Discussion}

Wang et al. reported that febuxostat pretreatment significantly attenuated murine myocardial ischemia/reperfusion injury in vivo as well as hypoxia/reoxygenation injury of rat cardiac myocytes in vitro [15]. The authors concluded that the protective effects of febuxostat against ischemia/reperfusion (hypoxia/reoxygenation) injury were due to the inhibition of ROS generation and mitochondrial apoptotic pathways. However, because allopurinol significantly improved the exercise tolerance in patients with angina pectoris [8], it seems that the protective effects of XO inhibitors were mainly due to the reduction in myocardial oxygen consumption rather than inhibition of oxidative stress-induced apoptosis. In the present study, we found no additive effects of inosine on myocardial ischemia/reperfusion injury. For human study, supplementation of inosine alone was reported to have no ergogenic effects on treadmill run and cycling performance $[13,16]$. Another study reported that inosine treatment had benefits in some patients with multiple sclerosis by raising serum uric acid levels and shifting the cytokine profile from Th1 to Th2 [17]. Because the XO enzyme activities in rodents were reported to be 100 times higher than that in humans [18], XO critically regulates the degradation of hypoxanthine and xanthine in rodents as compared with humans. This was supported by the findings of XO knockout mice whose blood and urinary concentrations of hypoxanthine and xanthine were markedly elevated than those of wild type. Therefore, XO inhibition alone seems to supply enough materials for ATP production pathways in rodents. This may be one of the reasons why there was no additive effects of inosine on rat myocardial ischemia/reperfusion injury in the present study. Whereas in humans, although administration of febuxostat alone minimally increased blood ATP/ADP ratios, febuxostat plus inosine administration clearly increased them [19]. We think the combination therapy with febuxostat plus inosine will be more effective than febuxostat alone in various disorders such as heart failure [5-7], multiple sclerosis [17], diabetes mellitus [20], angina pectoris [8], and especially myocardial ischemia/reperfusion injury. However, further clinical investigation is needed to verify this.

\section{References}

1. Opie LH (2012) Allopurinol for heart failure: novel mechanisms. J Am Coll Cardiol 59: 809-812. [Crossref]

2. Ekelund UEG, Harrison RW, Shokek O, Thakkar RN, Tunin RS, et al. (1999) Intravenous allopurinol decreases myocardial oxygen consumption and increases mechanical efficiency in dogs with pacing-induced heart failure. Circ Res 85: 437-445. [Crossref]

3. Ukai T, Cheng CP, Tachibana H, Igawa A, Zhang ZS, et al. (2001) Allopurinol enhances the contractile response to dobutamine and exercise in dogs with pacing-induced heart failure. Circulation 103: 750-755. [Crossref]

4. Stull LB, Leppo MK, Szweda L, Gao WD, Marba'n E (2004) Chronic treatment with allopurinol boosts survival and cardiac contractility in murine postischemic cardiomyopathy. Circ Res 95: 1005-1011. [Crossref]

5. Cappola TP, Kass DA, Nelson GS, Berger RD, Rosas GO, et al. (2001) Allopurinol improves myocardial efficiency in patients with idiopathic dilated cardiomyopathy. Circulation 104: 2407-2411. [Crossref]

6. Hirsch GA, Bottomley PA, Gerstenblith G, Weiss RG (2012) Allopurinol acutely increases adenosine triphospate energy delivery in failing human hearts. $J$ Am Coll Cardiol 59: 802-808. [Crossref]

7. Erdogan D, Tayyar S, Uysal BA, Icli A, Karabacak M, et al. (2012) Effects of allopurinol on coronary microvascular and left ventricular function in patients with idiopathic dilated cardiomyopathy. Can J Cardiol 28: 721-727.

8. Noman A, Ang DS, Ogston S, Lang CC, Struthers AD (2010) Effect of high-dose allopurinol on exercise in patients with chronic stable angina: a randomised, placebo controlled crossover trial. Lancet 375: 2161-2167. [Crossref] 
9. Nakagomi A, SaikinY, Noma S, Kohashi K, Morisawa T, et al. (2015) Effects of febuxostat and allopurinol on the inflammation and cardiacfunction in chronic heart failure patients with hyperuricemia. IJC Metab Endo 8: 46-55.

10. Aviado DM (1983) Inosine: a naturally occurring cardiotonic agent. J Pharmacol 14 Suppl 3: 47-71. [Crossref]

11. Czarnecki W, Czarnecki A (1989) Haemodynamic effects of inosine. A new drug for failing human heart? Pharmacol Res 21: 587-594. [Crossref]

12. McNaughton L, Dalton B, Tarr J (1999) Inosine supplementation has no effect on aerobic or anaerobic cycling performance. Int J Sport Nutr 9: 333-344. [Crossref]

13. Williams MH, Kreider RB, Hunter DW, Somma CT, Shall LM, et al. (1990) Effect of inosine supplementation on 3-mile treadmill run performance and $\mathrm{VO} 2$ peak. $\mathrm{Med} \mathrm{Sci}$ Sports Exerc 22: 517-522. [Crossref]

14. Seko Y, Enokawa Y, Tamatani T, Kannagi R, Yagita H, et al. (1996) Expression of sialyl LewisX in rat heart with ischemia/reperfusion and reduction of myocardial reperfusion injury by a monoclonal antibody against sialyl LewisX. $J$ Pathol $180: 305-$ 310. [Crossref]
15. Wang S, Li Y, Song X, Wang X, Zhao C, et al. (2015) Febuxostat pretreatment attenuates myocardial ischemia/reperfusion injury via mitochondrial apoptosis. $J$ Transl Med 13: 209. [Crossref]

16. McNaughton L, Dalton B, Tarr J (1999) Inosine supplementation has no effect on aerobic or anaerobic cycling performance. Int J Sport Nutr 9: 333-344. [Crossref]

17. Markowitz CE, Spitsin S, Zimmerman V, Jacobs D, Udupa JK, et al. (2009) The treatment of multiple sclerosis with inosine. J Altern Complement Med 15: 619-625. [Crossref]

18. Muxfeldt M, Schaper W (1987) The activity of xanthine oxidase in heart of pigs, guinea pigs, rabbits, rats, and humans. Basic Res Cardiol 82: 486-492. [Crossref]

19. Nishino T, Kamatani N (2017) Agent for enhancing ATP in cells. (WO2017033963) (in Japanese)

20. Mabley JG, Rabinovitch A, Suarez-Pinzon W, Haskó G, Pacher P, et al. (2003) Inosine protects against the development of diabetes in multiple-low-dose streptozotocin and nonobese diabetic mouse models of type 1 diabetes. Mol Med 9: 96-104. [Crossref]

Copyright: (2017 Yao T. This is an open-access article distributed under the terms of the Creative Commons Attribution License, which permits unrestricted use, distribution, and reproduction in any medium, provided the original author and source are credited. 